\title{
Enantioselective Enol Lactone Synthesis under Double Catalytic Conditions
}

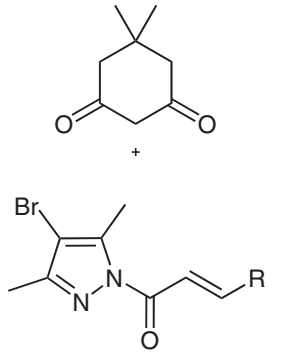

$(R, R)-\mathrm{DBFOX} / \mathrm{Ph}(10 \mathrm{~mol} \%)$ $\mathrm{Ni}\left(\mathrm{ClO}_{4}\right)_{2} \cdot 6 \mathrm{H}_{2} \mathrm{O}(10 \mathrm{~mol} \%)$

TMP (10 mol\%)

$\mathrm{Ac}_{2} \mathrm{O}$ (1.1 equiv)

THF, r.t.

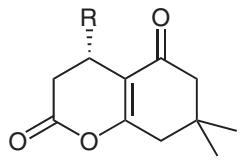

0 examples yields $53-99 \%$ $\%$ ee $=89-99 \%$

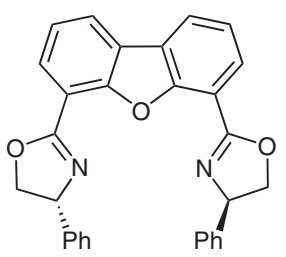

$(R, R)-\mathrm{DBFOX} / \mathrm{Ph}$

$\mathrm{R}=$ alkyl, aryl

Significance: Under the 'double-catalytic condition' protocol using the $(R, R)$-DBFOX/Ph complex of nickel(II) perchlorate hexahydrate and tetramethyl-piperidine, enol lactones were formed with high enantioselectivity. The mechanism proceeds via an enantioselective Michael addition followed by enolization of the diketone moiety, then cyclization with concomitant displacement of the pyrazole auxiliary. Catalyst loadings can be reduced to 2 mol\% and use of $\beta$-hydroxy-lactones instead of the diketone is also reported.
Comment: Enol lactones are found in many biologically active compounds such as coumarins and flavanoids, but synthetic methods for these targets are limited. A mild, highly enantioselective and efficient reaction has been developed to create substituted enol lactones. Using the 'doublecatalytic conditions', the chiral catalyst controls the enantioselectivity of the Michael addition and also activates the pyrazole leaving group to facilitate the relatively difficult lactonization step. The yields of the reaction are sensitive to the steric hindrance of the double bond although the ee's remain very high.
Metal-Catalyzed

Asymmetric

Synthesis and

Stereoselective

Reactions

Key Words

enol lactones Michael addition double-catalytic conditions

enantioselective reactions 